\title{
Magnitude of Anemia and Hematological Predictors among Children under 12 Years in Odisha, India
}

\author{
Shuchismita Behera and Gandham Bulliyya \\ Regional Medical Research Centre, Indian Council of Medical Research, Bhubaneswar 751023, India \\ Correspondence should be addressed to Gandham Bulliyya; gbrmrcicmr@gmail.com \\ Received 30 December 2015; Revised 11 March 2016; Accepted 16 March 2016 \\ Academic Editor: Aurelio Maggio
}

Copyright (C) 2016 S. Behera and G. Bulliyya. This is an open access article distributed under the Creative Commons Attribution License, which permits unrestricted use, distribution, and reproduction in any medium, provided the original work is properly cited.

Background. Anemia is a wide spread public health problem in India which affects children. The present study evaluates the prevalence of anemia and status of various hematological parameters among children of Khurda district, Odisha. Method. A total of 313 children aged $0-12$ years were enrolled for the study which included preschool ( $0-5$ years) and school aged (6-12 years) groups. Hematological indicators were measured by standard procedures, which include red blood cell (RBC) indicators, white blood cell (WBC) indicators, and plasma ferritin. Results. Mean hemoglobin ( $\mathrm{Hb}$ ) of the study population was $10.43 \pm 3.33 \mathrm{~g} / \mathrm{dL}$ and prevalence of anemia was $62 \%$. In this population, boys had a lower mean $\mathrm{Hb}$ value than that of the girls. All grades of anemia were higher among school age children than preschool children. Mean plasma ferritin was found to be higher in school age boys than their counterpart girls. The mean level of WBC count was found to be higher among preschool age boys than among the school age boys $(p=0.025)$. Conclusion. The prevalence of anemia was higher with concomitant acute infection among study population, which is a matter of concern. Since the hematological parameters are interrelated with each other as well as with the age and gender, relevant intervention strategy and constant monitoring are needed while providing public health nutrition programs to eradicate anemia.

\section{Introduction}

Anemia is a widespread public health problem associated with an increased risk of morbidity and mortality, especially in pregnant women and young children [1]. Globally 1.62 billion people are anemic, while among the preschool children the prevalence of anemia is $47.4 \%$. Nutritional anemia in South Asia accounts for nearly half of global cases of anemia. In India, anemia continues to be the major health problem in young children, adolescent girls, and pregnant women. Approximately $50 \%$ of the population suffers from nutritional anemia as known in countries where meat consumption is low [2].

In India, about 89 million children are anemic. The prevalence of anemia was $70 \%$ in children aged 6-59 months [3]. The highest prevalence of anemia was seen in children $<10$ years, especially in those $<5$ years [4]. Iron deficiency is one of the most common causes of anemia [5]. Besides iron, other nutrients such as vitamins $\mathrm{A}, \mathrm{E}$, and $\mathrm{C}$ also play key role in formation and protection of red blood cell (RBC) by stimulating stem cells as well as by activating a number of antioxidant enzymes [6]. Therefore inadequacy of any of these micronutrients may lead to anemia in the vulnerable sections of population. Studies have shown that preschool children are more vulnerable to the risk of iron deficiency anemia. The prevalence of iron deficiency anemia is the highest among preschool children. In this age group (6-59 months), body grows rapidly and requires high-iron-rich and nutritious food that may not be fulfilled by their normal diet. Low economic status, less education, and poor health of mothers due to meager dietary intake are the main causes of anemia. Anemia is the most predominant factor for morbidity and child mortality, and, hence, it is a critical health issue for children in India. Iron deficiency affects cognitive and motor development and increases susceptibility to infections. The prevention as well as timely management of anemia is essential to attain Sustainable Development Goal-3 (SDG) on ensuring healthy lives and promoting wellbeing for all at all 
ages. Further actions are required to reach the World Health Assembly target of a $50 \%$ reduction of anemia in women of reproductive age by 2025 .

Odisha (formerly Orissa) is one of eight empowered action group (EAG) states of India with poor demographic and socioeconomic indicators including maternal and child health. The Clinical, Anthropometric, and Biochemical (CAB) survey conducted recently in 2014 shows that $70.6 \%$ and $81.2 \%$ of children aged 6-59 months and 5-9-yearold children are suffering from anemia. Hence, information regarding young children is inadequate on factors affecting anemia. However, no report is available on prevalence of anemia among children in Khurda district. In the present study, an attempt has been made to assess the magnitude of iron deficiency anemia by measuring hematological indices.

\section{Materials and Methods}

2.1. Setting. The study was conducted in the rural surroundings of Bhubaneswar city in Khurda district, the state capital of Odisha located on the east coast of India, by the Bay of Bengal. Apparently healthy children aged less than 12 years were chosen for the study, which included preschool children ( $0-5$ years) and school age children (6-12 years). Children having medication in the past fortnight prior to data collection and unwilling individuals were excluded from the study.

2.2. Study Design. This is a cross-sectional community-based survey. All children and their parents were informed about the purpose and the method of the research and the voluntary nature of participation in the study verbally and in written form.

2.3. Ethical Consideration. Informed written consent was obtained from the parents of each child after the study objective was explained. The study protocol was approved by the Institutional Human Ethical Committee of Regional Medical Research Centre, Bhubaneswar.

2.4. Data Collection. A pretested questionnaire was applied to obtain relevant information of demographic and socioeconomic data. Age of each child was collected from date of birth certificate or birth records available with mother. Confirmation of a child's age was made with the mother with the help of Anganwadi Workers, community health workers.

2.5. Anthropometric Measurements. Body weight and height were measured using standardised equipment and procedures. Body mass index (BMI) for each child was calculated based on the ratio of weight $(\mathrm{kg})$ to height in square meters. BMI data were transformed to $z$-scores, namely, BMI-for-age $z$-score (BAZ) using the WHO Growth Standards [7].

2.6. Blood Samples. Either finger prick or venous blood was collected according to the agreement of the participants. The finger prick blood was transferred to Whatman number 1 filter paper while two $\mathrm{mL}$ of venous blood was dispensed into
TABLE 1: Hemoglobin concentrations ( $\mathrm{g} / \mathrm{dL}$ ) for the diagnosis of anemia and assessment of severity according to the WHO/UNICEF/ UNU (2001).

\begin{tabular}{lcccc}
\hline \multicolumn{5}{c}{ Anemia measured by hemoglobin $(\mathrm{g} / \mathrm{dL})$} \\
& Anemia & Mild & Moderate & Severe \\
\hline Children 6-59 months & $<11.0$ & $10-10.9$ & $7.0-9.9$ & $<7.0$ \\
Children 5-11 years & $<11.5$ & $10-11.4$ & $7.0-9.9$ & $<7.0$ \\
Children 12-14 years & $<12.0$ & $10-11.9$ & $7.0-9.9$ & $<7.0$ \\
\hline
\end{tabular}

vials containing EDTA anticoagulant bottles. Hematological and biochemical investigations were carried out in nutrition laboratory.

2.7. Biochemical Estimations. Venous blood was subjected to complete blood count (CBC), which was performed by an automated analyzer MS4 (Melet Schloesing 4, Germany) used for the in vitro diagnostic testing. The blood was well mixed (though not shaken) and placed on a rack in the analyzer. The instrument counted the number and type of different cells within the blood and results were printed out that included hemoglobin $(\mathrm{Hb})$, red blood cell (RBC), hematocrit (HCT), mean corpuscular volume (MCV), mean corpuscular hemoglobin $(\mathrm{MCH})$, mean corpuscular hemoglobin concentration (MCHC), red cell distribution width (RDW), white blood cell (WBC), granulocyte, thrombocyte (platelet), lymphocyte, and monocytes. Adequate quality control measures were taken on each test procedure to ensure the reliability of the results. The validity of $\mathrm{Hb}$ measure was confirmed by checking the reproducibility of the results of sample aliquots by cyanmethemoglobin method. The finger prick blood was used to estimate hemoglobin $(\mathrm{Hb})$ by cyanmethemoglobin method [8]. Plasma ferritin was estimated using ELISA kits obtained from United Biotech Inc. Magiwell Ferritin, USA (K951993).

2.8. Statistical Analysis. Anemia was defined as $\mathrm{Hb}$ concentration $<11 \mathrm{~g} / \mathrm{dL}$ for children aged between 6 and 59 months while $<11.5 \mathrm{~g} / \mathrm{dL}$ for children aged between 5 and 11 years and $<12 \mathrm{~g} / \mathrm{dL}$ for children aged 12 years according to WHO as shown in Table 1 [9]. Further, normal reference ranges used for hematological indicators (red blood indices and white blood indices) are provided in Table $2[10,11]$. Data were entered in Microsoft Excel 2007 and all statistical analyses were performed with GraphPad Prism software (version 4.00). SPSS for Windows version 17.0, Chicago, USA, was also used for data analysis. Descriptive characteristics (mean and standard deviation) and percentage were performed for each parameter separately. Chi-square and independent $t$-test were used for proportions and mean comparisons between groups, respectively. Pearson's correlation tests were performed to examine the relationships between hematological indicators. The strength of association is measured by unadjusted odds ratio (OR) and 95\% confidence interval (CI).

\section{Results}

A total of 313 children provided blood samples for estimation of $\mathrm{Hb}$, ferritin (250), hematological indicators (139), and 
TABLE 2: Reference range/cut-off values assigned for different hematological parameters.

\begin{tabular}{|c|c|c|c|}
\hline Hematological parameters & Age group & Reference value & Reference \\
\hline \multirow{4}{*}{ Red blood count (million $/ \mathrm{mm}^{3}$ ) } & 6 months -2 years & $3.7-5.3$ & \multirow{24}{*}[10]{} \\
\hline & & & \\
\hline & 2 years -6 years & $3.9-5.3$ & \\
\hline & 6 years- 12 years & $4.0-5.2$ & \\
\hline \multirow{3}{*}{ Hematocrit (\%) } & 6 months -2 years & $33-39$ & \\
\hline & 2 years -6 years & $34-40$ & \\
\hline & 6 years -12 years & $35-45$ & \\
\hline \multirow{3}{*}{ Mean corpuscular volume (fl) } & 6 months -2 years & $70-86$ & \\
\hline & 2 years -6 years & $75-87$ & \\
\hline & 6 years-12 years & $77-95$ & \\
\hline \multirow{3}{*}{ Mean corpuscular hemoglobin (pg) } & 6 months -2 years & $23-31$ & \\
\hline & 2 years -6 years & $24-30$ & \\
\hline & 6 years -12 years & $25-33$ & \\
\hline \multirow{2}{*}{$\begin{array}{l}\text { Mean corpuscular hemoglobin } \\
\text { concentration }(\mathrm{g} / \mathrm{dL})\end{array}$} & 6 months -2 years & $30.0-36.0$ & \\
\hline & 2 years + & $32.3-35.7$ & \\
\hline \multirow{4}{*}{$\begin{array}{l}\text { White blood counts } \\
\text { (thousand } / \mathrm{mm}^{3} \text { ) }\end{array}$} & 6 months -2 years & $6.0-17.0$ & \\
\hline & 2 years -4 years & $6.0-15.5$ & \\
\hline & 4 years -6 years & $5.5-14.5$ & \\
\hline & 6 years- -12 years & $4.5-13.5$ & \\
\hline \multirow{5}{*}{ Thrombocytes (thousand $/ \mathrm{mm}^{3}$ ) } & 0-1 month & $250-450$ & \\
\hline & 1 month-1 year & $300-750$ & \\
\hline & $1-3$ years & $250-600$ & \\
\hline & $3-7$ years & $250-550$ & \\
\hline & $7-12$ years & $200-450$ & \\
\hline Granulocytes (\%) & All age groups & $18-45$ & \multirow{4}{*}[11]{} \\
\hline Monocytes (\%) & All age groups & $4-11$ & \\
\hline Lymphocytes (\%) & All age groups & $45-75$ & \\
\hline Red cell distribution width (\%) & All age groups & $11-15$ & \\
\hline
\end{tabular}

WBC differential count (131). The mean characteristics of BAZ and hematological parameters are shown in Table 3. The mean BAZ of study population was $-1.04 \pm 1.53$ and no significant difference was observed between the age or gender groups. The mean $\mathrm{Hb}$ of the study population was $10.43 \pm 3.33 \mathrm{~g} / \mathrm{dL}$. The mean $\mathrm{Hb}$ among preschool children was $10.45 \pm 2.99 \mathrm{~g} / \mathrm{dL}$ of which boys had $10.57 \pm 3.01 \mathrm{~g} / \mathrm{dL}$ and girls had $10.27 \pm 2.99 \mathrm{~g} / \mathrm{dL}$. The mean $\mathrm{Hb}$ among school age children was found to be $10.42 \pm 3.491 \mathrm{~g} / \mathrm{dL}$ of which boys had $9.78 \pm 3.96 \mathrm{~g} / \mathrm{dL}$ and girls had $10.85 \pm 3.98 \mathrm{~g} / \mathrm{dL}$. Hb, HCT, MCV, and MCH of school age boys were significantly lower than girls ( $p=0.029,0.042,0.0002$, and 0.023 , resp.). The mean ferritin level among boys was significantly higher than girls $(p=0.0002)$, which was chiefly exhibited by school age children. The mean WBC count was found to be higher among preschool boys than among school age boys $(p=0.025)$. Mean MCV and MCH were significantly higher among school age girls than preschool girls $(p=0.026$ and 0.011, resp.). Also, $\mathrm{MCH}$ and $\mathrm{MCHC}$ of preschool children were low compared to school children $(p=0.009$ and 0.006 , resp.). Mean RDW of preschool girls was higher than their male counterpart as well as school age girls $(p=0.016$ and 0.026).

The prevalence of different grades of anemia according to $\mathrm{Hb}$ level is depicted in Table 4. Overall occurrence of anemia was $62 \%$ comprised of $23 \%$ mild, $23 \%$ moderate, and $16 \%$ severe categories. School age children were found to be more anemic than preschool children. Prevalence of anemia was $48.5 \%$ among preschool children of which $47.6 \%$ were boys and $50.0 \%$ were girls. Mild, moderate, and severe anemia were found to be $12.9 \%, 22.7 \%$, and $12.9 \%$, respectively. Among school age children $68.9 \%$ had anemia, of which $27.4 \%$ were mildly, $23.6 \%$ were moderately, and $17.9 \%$ were severely anemic. Girls were significantly more anemic than boys in the age group of $11-12$ years (93.1\% versus $72.2 \%$, $p=0.028)$ while more boys were anemic in the age group 10 - 11 years $(84.6 \%$ versus $53.1 \%, p=0.048$ ).

Table 5 shows the hematological indicators for anemia with standard reference ranges. Deficiency of RBC count was found to be $47.5 \%$ in children indicating iron, vitamin B12, or folate deficiency or hemolysis. Further, deficiency of HCT was 52.5\% indicating the same. Deficiency of MCV, 
TABLE 3: Mean BMI-for-age $z$-score and hematological parameters among children in Odisha, India.

\begin{tabular}{|c|c|c|c|c|c|c|c|}
\hline Variables & Sex & $N$ & Preschool children & $N$ & School age children & $N$ & Pooled children \\
\hline \multirow{3}{*}{ BMI-for-age $z$-score } & Boys & 63 & $-0.96 \pm 1.82$ & 85 & $-0.96 \pm 1.59$ & 148 & $-0.96 \pm 1.68$ \\
\hline & Girls & 38 & $-0.87 \pm 1.34$ & 127 & $-1.18 \pm 1.37$ & 165 & $-1.11 \pm 1.368$ \\
\hline & Total & 101 & $-0.93 \pm 1.65$ & 212 & $-1.09 \pm 1.46$ & 313 & $-1.04 \pm 1.53$ \\
\hline \multirow{3}{*}{ Hemoglobin (g/dL) } & Boys & 63 & $10.57 \pm 3.01$ & 85 & $9.78 \pm 3.96$ & 148 & $10.11 \pm 3.59$ \\
\hline & Girls & 38 & $10.27 \pm 2.99$ & 127 & $10.85 \pm 3.09^{*}$ & 165 & $10.71 \pm 3.06$ \\
\hline & Total & 101 & $10.45 \pm 2.99$ & 212 & $10.42 \pm 3.49$ & 313 & $10.43 \pm 3.33$ \\
\hline \multirow{3}{*}{ Plasma ferritin (ng/mL) } & Boys & 49 & $177.9 \pm 226.8$ & 75 & $279.5 \pm 280.0^{\$}$ & 124 & $239.4 \pm 264.0$ \\
\hline & Girls & 23 & $162.1 \pm 218.2$ & 103 & $122.8 \pm 176.3^{*}$ & 126 & $130.0 \pm 184.4^{*}$ \\
\hline & Total & 72 & $172.9 \pm 222.6$ & 178 & $189.2 \pm 238.4$ & 250 & $184.5 \pm 233.6$ \\
\hline \multirow{3}{*}{$\begin{array}{l}\text { White blood cell count } \\
\text { (thousand } / \mathrm{mm}^{3} \text { ) }\end{array}$} & Boys & 29 & $9.51 \pm 6.43$ & 51 & $6.72 \pm 4.44^{\$}$ & 80 & $7.74 \pm 5.38$ \\
\hline & Girls & 17 & $8.12 \pm 5.45$ & 42 & $8.03 \pm 7.13$ & 59 & $8.05 \pm 6.64$ \\
\hline & Total & 46 & $8.99 \pm 6.06$ & 93 & $7.31 \pm 5.81$ & 139 & $7.87 \pm 5.93$ \\
\hline \multirow{3}{*}{$\begin{array}{l}\text { Red blood cell count } \\
\left(\mathrm{million} / \mathrm{mm}^{3}\right)\end{array}$} & Boys & 29 & $4.21 \pm 1.16$ & 51 & $3.69 \pm 1.29$ & 80 & $3.88 \pm 1.26$ \\
\hline & Girls & 17 & $3.823 \pm 1.71$ & 42 & $4.02 \pm 1.59^{*}$ & 59 & $3.96 \pm 1.61$ \\
\hline & Total & 46 & $4.07 \pm 1.38$ & 93 & $3.84 \pm 1.43$ & 139 & $3.92 \pm 1.41$ \\
\hline \multirow{3}{*}{ Hematocrit (\%) } & Boys & 29 & $35.20 \pm 10.25$ & 51 & $29.94 \pm 10.93^{\$}$ & 80 & $31.85 \pm 10.93$ \\
\hline & Girls & 17 & $29.52 \pm 12.60$ & 42 & $35.33 \pm 14.26^{*}$ & 59 & $33.65 \pm 13.95$ \\
\hline & Total & 46 & $33.10 \pm 11.38$ & 93 & $32.37 \pm 12.76$ & 139 & $32.61 \pm 12.28$ \\
\hline \multirow{3}{*}{$\begin{array}{l}\text { Mean corpuscular } \\
\text { volume (fl) }\end{array}$} & Boys & 29 & $83.58 \pm 6.96$ & 51 & $81.35 \pm 8.11$ & 80 & $82.16 \pm 7.74$ \\
\hline & Girls & 17 & $81.15 \pm 14.09$ & 42 & $88.01 \pm 8.61^{* \$}$ & 59 & $86.04 \pm 10.82^{*}$ \\
\hline & Total & 46 & $82.68 \pm 10.11$ & 93 & $84.36 \pm 8.94$ & 139 & $83.81 \pm 9.34$ \\
\hline \multirow{3}{*}{$\begin{array}{l}\text { Mean corpuscular } \\
\text { hemoglobin (pg) }\end{array}$} & Boys & 29 & $25.41 \pm 2.72$ & 51 & $26.18 \pm 3.63$ & 80 & $25.90 \pm 3.33$ \\
\hline & Girls & 17 & $24.76 \pm 5.32$ & 42 & $27.95 \pm 3.73^{* \$}$ & 59 & $27.03 \pm 4.44$ \\
\hline & Total & 46 & $25.17 \pm 3.84$ & 93 & $26.98 \pm 3.76^{\$}$ & 139 & $26.38 \pm 3.87$ \\
\hline \multirow{3}{*}{$\begin{array}{l}\text { Mean corpuscular } \\
\text { hemoglobin } \\
\text { concentration }(\mathrm{g} / \mathrm{dL})\end{array}$} & Boys & 29 & $30.48 \pm 2.35$ & 51 & $32.29 \pm 3.39^{\$}$ & 80 & $31.63 \pm 3.16$ \\
\hline & Girls & 17 & $30.89 \pm 2.83$ & 42 & $31.82 \pm 2.52$ & 59 & $31.55 \pm 2.62$ \\
\hline & Total & 46 & $30.63 \pm 2.52$ & 93 & $32.08 \pm 3.02^{\$}$ & 139 & $31.60 \pm 2.93$ \\
\hline \multirow{3}{*}{$\begin{array}{l}\text { Red cell distribution } \\
\text { width }(\%)\end{array}$} & Boys & 29 & $10.79 \pm 1.56$ & 51 & $11.73 \pm 3.00$ & 80 & $11.39 \pm 2.60$ \\
\hline & Girls & 17 & $13.28 \pm 5.04^{*}$ & 42 & $11.01 \pm 2.58^{\$}$ & 59 & $11.66 \pm 3.58$ \\
\hline & Total & 46 & $11.71 \pm 3.47$ & 93 & $11.40 \pm 2.83$ & 139 & $11.50 \pm 3.05$ \\
\hline \multirow{3}{*}{ Granulocyte (\%) } & Boys & 29 & $36.40 \pm 17.49$ & 48 & $38.12 \pm 17.28$ & 77 & $37.49 \pm 17.26$ \\
\hline & Girls & 17 & $35.76 \pm 18.35$ & 37 & $35.54 \pm 17.52$ & 54 & $35.61 \pm 17.61$ \\
\hline & Total & 46 & $36.16 \pm 17.61$ & 85 & $37.00 \pm 17.33$ & 131 & $36.71 \pm 17.37$ \\
\hline \multirow{3}{*}{ Monocyte (\%) } & Boys & 29 & $6.76 \pm 4.75$ & 48 & $5.44 \pm 4.02$ & 77 & $5.92 \pm 4.32$ \\
\hline & Girls & 17 & $4.81 \pm 2.30$ & 37 & $5.59 \pm 3.47$ & 54 & $5.34 \pm 3.15$ \\
\hline & Total & 46 & $6.02 \pm 4.08$ & 85 & $5.50 \pm 3.77$ & 131 & $5.68 \pm 3.87$ \\
\hline \multirow{3}{*}{ Lymphocyte (\%) } & Boys & 29 & $55.88 \pm 15.28$ & 48 & $56.45 \pm 17.58$ & 77 & $56.24 \pm 16.67$ \\
\hline & Girls & 17 & $59.22 \pm 17.62$ & 37 & $58.87 \pm 17.34$ & 54 & $58.98 \pm 17.26$ \\
\hline & Total & 46 & $57.14 \pm 16.09$ & 85 & $57.50 \pm 17.41$ & 131 & $57.38 \pm 16.91$ \\
\hline \multirow{3}{*}{$\begin{array}{l}\text { Thrombocyte count } \\
\text { (thousand } / \mathrm{mm}^{3} \text { ) }\end{array}$} & Boys & 29 & $331.2 \pm 450.6$ & 48 & $229.9 \pm 233.3$ & 77 & $267.7 \pm 332.2$ \\
\hline & Girls & 17 & $222.2 \pm 164.1$ & 37 & $205.4 \pm 149.2$ & 54 & $210.7 \pm 152.7$ \\
\hline & Total & 46 & $290.0 \pm 370.5$ & 85 & $219.1 \pm 199.9$ & 131 & $243.8 \pm 272.5$ \\
\hline
\end{tabular}

${ }^{*} p<0.05$ for boys versus girls; ${ }^{\$} p<0.05$ for preschool versus school children. 
TABLE 4: Prevalence (\%) of anemia by hemoglobin ( $\mathrm{g} / \mathrm{dL})$ among children in Odisha.

\begin{tabular}{|c|c|c|c|c|c|c|c|c|c|}
\hline \multirow{2}{*}{$\begin{array}{l}\text { Age } \\
\text { group (years) }\end{array}$} & \multirow{2}{*}{ Sex } & \multirow{2}{*}{$N$} & \multicolumn{4}{|c|}{ Anemia grade } & \multirow{2}{*}{ Total anemic } & \multirow{2}{*}{$p$ value } & \multirow{2}{*}{ OR (95\% CI) } \\
\hline & & & Normal & Mild & Moderate & Severe & & & \\
\hline \multirow{3}{*}{ Preschool children } & Boys & 63 & $52.4(33)$ & $12.7(8)$ & $22.2(14)$ & $12.7(8)$ & $47.6(30)$ & \multirow{3}{*}{0.816} & \multirow{3}{*}{$1.10(0.49-2.46)$} \\
\hline & Girls & 38 & $50.0(19)$ & $13.2(5)$ & $23.6(9)$ & $13.2(5)$ & $50.0(19)$ & & \\
\hline & Total & 101 & $51.5(52)$ & $12.9(13)$ & $22.7(23)$ & $12.9(13)$ & $48.5(49)$ & & \\
\hline \multirow{3}{*}{ School age children } & Boys & 85 & $27.1(23)$ & $17.6(15)$ & $25.9(22)$ & $29.4(25)$ & $72.9(62)$ & \multirow{3}{*}{0.295} & \multirow{3}{*}{$1.38(0.75-2.52)$} \\
\hline & Girls & 127 & $33.9(43)$ & $33.9(43)$ & $22.0(28)$ & $10.2(13)$ & $66.1(84)$ & & \\
\hline & Total & 212 & $31.1(66)$ & $27.4(58)$ & $23.6(50)$ & $17.9(38)$ & $68.9(146)$ & & \\
\hline \multirow{3}{*}{ Pooled children } & Boys & 148 & $37.8(56)$ & $15.6(23)$ & $24.3(36)$ & $22.3(33)$ & $62.2(92)$ & \multirow{3}{*}{0.962} & \multirow{3}{*}{$1.01(0.64-1.59)$} \\
\hline & Girls & 165 & $37.6(62)$ & $29.1(48)$ & $22.4(37)$ & $10.9(18)$ & $62.4(103)$ & & \\
\hline & Total & 313 & 37.7 (118) & $22.7(71)$ & $23.3(73)$ & $16.3(51)$ & $62.3(195)$ & & \\
\hline
\end{tabular}

$\mathrm{OR}=$ odds ratio, $\mathrm{CI}=$ confidence interval; figures in parentheses are sample number.

TABLE 5: Distribution of hematological (red blood cell) indicators by age group and sex among children in Odisha, India.

\begin{tabular}{|c|c|c|c|c|c|c|c|c|c|c|}
\hline \multirow{2}{*}{ Parameter } & \multirow{2}{*}{ Category } & \multicolumn{3}{|c|}{ Preschool children } & \multicolumn{3}{|c|}{ School age children } & \multicolumn{3}{|c|}{ Pooled children } \\
\hline & & Boys & Girls & Total & Boys & Girls & Total & Boys & Girls & Total \\
\hline \multirow{2}{*}{$\begin{array}{l}\text { Red blood cell count } \\
\left(\mathrm{million} / \mathrm{mm}^{3}\right)\end{array}$} & Normal & $75.9(22)$ & $47.1(8)$ & $65.2(30)$ & $47.1(24)$ & $45.2(19)$ & $46.2(43)$ & $57.5(46)$ & $45.8(27)$ & $52.5(73)$ \\
\hline & Deficiency & $24.1(7)$ & $52.9(9)$ & $34.8(16)$ & $52.9(27)$ & $54.8(23)$ & $53.8(50)$ & $42.5(34)$ & $54.2(32)$ & $47.5(66)$ \\
\hline \multirow{2}{*}{ Hematocrit (\%) } & Normal & $65.5(19)$ & $47.1(8)$ & $58.7(27)$ & $35.3(18)$ & $50.0(21)$ & $41.9(39)$ & $46.2(37)$ & $49.2(29)$ & $47.5(66)$ \\
\hline & Deficiency & $34.5(10)$ & $52.9(9)$ & $41.3(19)$ & $64.7(33)$ & $50.0(21)$ & $58.1(54)$ & $53.8(43)$ & $50.8(30)$ & $52.5(73)$ \\
\hline \multirow{3}{*}{ Mean corpuscular volume (fl) } & High & $24.1(7)$ & $17.7(3)$ & $21.7(10)$ & $5.9(3)$ & $16.7(7)$ & $10.7(10)$ & $12.5(10)$ & $16.9(10)$ & $14.4(20)$ \\
\hline & Standard & $65.6(19)$ & $52.9(9)$ & $60.9(28)$ & $74.5(38)$ & $76.2(32)$ & $75.3(70)$ & $71.3(57)$ & $69.5(41)$ & $70.5(98)$ \\
\hline & Deficiency & $10.3(3)$ & $29.4(5)$ & $17.4(8)$ & $19.6(10)$ & $7.1(3)$ & $14.0(13)$ & $16.2(13)$ & $13.6(8)$ & $15.1(21)$ \\
\hline \multirow{3}{*}{$\begin{array}{l}\text { Mean corpuscular hemoglobin } \\
(\mathrm{pg})\end{array}$} & High & $3.5(1)$ & $17.6(3)$ & $8.7(4)$ & $3.9(2)$ & $4.8(2)$ & $4.3(4)$ & $5.0(4)$ & $6.8(4)$ & $5.7(8)$ \\
\hline & Standard & $75.8(22)$ & $41.2(7)$ & $63.0(29)$ & $54.9(28)$ & $73.8(31)$ & $63.4(59)$ & $61.2(49)$ & $66.1(39)$ & $63.4(88)$ \\
\hline & Deficiency & $20.7(6)$ & $41.2(7)$ & $28.3(13)$ & $41.2(21)$ & $21.4(9)$ & $32.3(30)$ & $33.8(27)$ & $27.1(16)$ & 30.9 (43) \\
\hline \multirow{2}{*}{$\begin{array}{l}\text { Mean corpuscular hemoglobin } \\
\text { concentration }(\mathrm{g} / \mathrm{dL})\end{array}$} & Normal & 37.9 (11) & $58.8(10)$ & $46.7(21)$ & $45.1(23)$ & $42.9(18)$ & $44.1(41)$ & $42.5(34)$ & $47.5(28)$ & $44.6(62)$ \\
\hline & Deficiency & $62.1(18)$ & $41.2(7)$ & $54.3(25)$ & $54.9(28)$ & $57.1(24)$ & $55.9(52)$ & $49.5(46)$ & $52.5(31)$ & $55.4(77)$ \\
\hline \multirow{3}{*}{ Red cell distribution width (\%) } & High & $3.5(1)$ & $29.4(5)$ & $13.1(6)$ & $11.8(6)$ & $9.5(4)$ & $10.8(10)$ & $8.8(7)$ & $15.3(9)$ & $11.5(16)$ \\
\hline & Standard & $24.1(7)$ & $41.2(7)$ & $30.4(14)$ & $35.3(18)$ & $19.1(8)$ & $27.9(26)$ & $31.2(25)$ & $25.4(15)$ & $28.8(40)$ \\
\hline & Deficiency & $72.4(21)$ & $29.4(5)$ & $56.5(26)$ & $52.9(27)$ & $71.4(30)$ & $61.3(57)$ & $60.0(48)$ & $59.3(35)$ & $59.7(83)$ \\
\hline
\end{tabular}

Normal $=$ number above the lower cut-off, High $=$ number above higher cut-off value, Standard $=$ number within the range, and Deficiency $=$ number below the lower cut-off level. Figures in parentheses are sample number.

$\mathrm{MCH}$, and $\mathrm{MCHC}$ was $15.1 \%, 30.9 \%$, and $55.4 \%$, respectively, demonstrating a probable iron deficiency while value above the reference level for $\mathrm{MCV}$ and $\mathrm{MCH}$ indicates probability of vitamin $\mathrm{B}_{12}$ deficiency for $14.4 \%$ and $5.7 \%$, respectively. Microcytic anemia in terms of MCV and $\mathrm{MCH}$ was higher among school age boys than girls. Although deficiency of RDW (<11\%) was $59.7 \%$, it does not signify to a concerning problem; however, a higher cut-off value indicates an iron/vitamin $\mathrm{B}_{12} /$ folate deficiency of $11.5 \%$ (RDW > 15\%).

Table 6 represents WBC indicators and thrombocyte according to standard reference ranges. The prevalence of WBC deficiency was $35.3 \%$ indicating immunosuppression/viral infection. About $12.2 \%$ of children had WBC level above the cut-off level to represent inflammation or infection. The prevalence of granulocyte, monocyte, and lymphocyte deficiency was $64.9 \%, 32.8 \%$, and $0.8 \%$, respectively, which is indicative of immunosuppression. High level of granulocyte was prevalent in $1.5 \%$ of children indicating infection/inflammation. About $6.9 \%$ of children had high monocyte count demonstrating chronic infection while $74.8 \%$ had high lymphocyte count reflecting their susceptibility to viral infections. About $63.4 \%$ of children were subclinically deficient for thrombocyte (platelet), which is an important bloodclotting factor. Higher level of thrombocytes observed in $7.6 \%$ of children is an indicative sign of viral infection/pernicious anemia.

The relationship between different health indicators of children is analyzed by correlation to establish the degree of association (Table 7). Hb, HCT, and RBC count were positively correlated with BAZ while the latter was negatively correlated with RDW. Hb was positively correlated with RBC, $\mathrm{HCT}, \mathrm{MCV}, \mathrm{MCH}$, and monocyte but inversely correlated 
TABLE 6: Distribution of hematological (white blood cell) indicators by age group and sex among children in Odisha, India.

\begin{tabular}{|c|c|c|c|c|c|c|c|c|c|c|}
\hline \multirow{2}{*}{ Parameter } & \multirow{2}{*}{ Category } & \multicolumn{3}{|c|}{ Preschool children } & \multicolumn{3}{|c|}{ School age children } & \multicolumn{3}{|c|}{ Pooled children } \\
\hline & & Boys & Girls & Total & Boys & Girls & Total & Boys & Girls & Total \\
\hline \multirow{3}{*}{ WBC (thousand $/ \mathrm{mm}^{3}$ ) } & High & $13.8(4)$ & $11.8(2)$ & $13.1(6)$ & $5.9(3)$ & $19.0(8)$ & $11.8(11)$ & $8.7(7)$ & $16.9(10)$ & $12.2(17)$ \\
\hline & Standard & $62.1(18)$ & $47.1(8)$ & $56.5(26)$ & $60.8(31)$ & $38.1(16)$ & $50.6(47)$ & $61.3(49)$ & $40.7(24)$ & $52.5(73)$ \\
\hline & Deficiency & $24.1(7)$ & $41.1(7)$ & $30.4(14)$ & $33.3(17)$ & $42.9(18)$ & $37.6(35)$ & $30.0(24)$ & $42.4(25)$ & $35.3(49)$ \\
\hline \multirow{3}{*}{ Granulocytes (\%) } & High & $3.4(1)$ & $0.0(0)$ & $2.2(1)$ & $2.1(1)$ & $0.0(0)$ & $1.2(1)$ & $2.6(2)$ & $0.0(0)$ & $1.5(2)$ \\
\hline & Standard & $34.5(10)$ & $35.3(6)$ & $34.8(16)$ & $39.6(19)$ & $24.3(9)$ & $32.9(28)$ & $37.7(29)$ & $27.8(15)$ & $33.6(44)$ \\
\hline & Deficiency & $62.1(18)$ & $64.7(11)$ & $63.0(29)$ & $58.3(28)$ & $75.7(28)$ & $65.9(56)$ & $59.7(46)$ & $72.2(39)$ & $64.9(85)$ \\
\hline \multirow{3}{*}{ Monocytes (\%) } & High & $13.8(4)$ & $0.0(0)$ & $8.7(4)$ & $6.3(3)$ & $5.4(2)$ & $5.9(5)$ & $9.1(7)$ & $3.7(2)$ & $6.9(9)$ \\
\hline & Standard & $58.6(17)$ & $64.7(11)$ & $60.9(28)$ & $56.3(27)$ & $64.9(24)$ & $60.0(51)$ & $57.1(44)$ & $64.8(35)$ & $60.3(79)$ \\
\hline & Deficiency & $27.6(8)$ & $35.3(6)$ & $30.4(14)$ & $37.4(18)$ & $29.7(11)$ & $34.1(29)$ & $33.8(26)$ & $31.5(17)$ & $32.8(43)$ \\
\hline \multirow{3}{*}{ Lymphocytes (\%) } & High & $69.0(20)$ & $70.6(12)$ & $69.6(32)$ & $77.1(37)$ & $78.4(29)$ & $77.6(66)$ & $74.0(57)$ & $75.9(41)$ & $74.8(98)$ \\
\hline & Standard & $31.0(9)$ & $29.4(5)$ & $30.4(14)$ & $20.8(10)$ & $21.6(8)$ & $21.2(18)$ & 24.7 (19) & $24.1(13)$ & $24.4(32)$ \\
\hline & Deficiency & $0.0(0)$ & $0.0(0)$ & $0.0(0)$ & $2.1(1)$ & $0.0(0)$ & $1.2(1)$ & $1.3(1)$ & $0.0(0)$ & $0.8(1)$ \\
\hline \multirow{3}{*}{$\begin{array}{l}\text { Thrombocytes } \\
\text { (thousand } / \mathrm{mm}^{3} \text { ) }\end{array}$} & High & $10.3(3)$ & $5.9(1)$ & $8.7(4)$ & $10.4(5)$ & $2.7(1)$ & $7.1(6)$ & $10.5(8)$ & $3.7(2)$ & $7.6(10)$ \\
\hline & Standard & $31.0(9)$ & $35.3(6)$ & $32.6(15)$ & $22.9(11)$ & $32.4(12)$ & $27.0(23)$ & $25.9(20)$ & $33.3(18)$ & $29.0(38)$ \\
\hline & Deficiency & $58.7(17)$ & $58.8(10)$ & $58.7(27)$ & $66.7(32)$ & $64.9(24)$ & $65.9(56)$ & $64.5(49)$ & $63.0(34)$ & $63.4(83)$ \\
\hline
\end{tabular}

Normal $=$ number above the lower cut-off, High $=$ number above higher cut-off value, Standard $=$ number within the range, and Deficiency $=$ number below the lower cut-off level. Figures in parentheses are sample number.

TABLE 7: Pearson's correlation coefficients $(r)$ of different parameters in children of Odisha, India.

\begin{tabular}{|c|c|c|c|c|c|c|c|c|c|c|c|c|}
\hline & BAZ & $\mathrm{Hb}$ & WBC & RBC & HCT & $\mathrm{MCV}$ & $\mathrm{MCH}$ & $\mathrm{MCHC}$ & THR & RDW & GRA & MON \\
\hline BAZ & 1.00 & & & & & & & & & & & \\
\hline $\mathrm{Hb}$ & $0.381^{\ddagger}$ & 1.000 & & & & & & & & & & \\
\hline WBC & 0.454 & $-0.232^{\dagger}$ & 1.000 & & & & & & & & & \\
\hline $\mathrm{RBC}$ & $0.288^{\ddagger}$ & $0.871^{\ddagger}$ & -0.158 & 1.000 & & & & & & & & \\
\hline $\mathrm{HCT}$ & $0.268^{\dagger}$ & $0.943^{\ddagger}$ & $-0.199^{*}$ & $0.940^{\ddagger}$ & 1.000 & & & & & & & \\
\hline $\mathrm{MCV}$ & -0.041 & $0.337^{\ddagger}$ & -0.079 & -0.019 & $0.281^{*}$ & 1.000 & & & & & & \\
\hline $\mathrm{MCH}$ & -0.032 & $0.209^{*}$ & -0.093 & $-0.189^{*}$ & 0.066 & $0.757^{\ddagger}$ & 1.000 & & & & & \\
\hline $\mathrm{MCHC}$ & -0.003 & -0.006 & 0.133 & -0.043 & -0.024 & 0.049 & 0.089 & 1.000 & & & & \\
\hline THR & 0.057 & -0.052 & $0.373^{\ddagger}$ & -0.033 & -0.064 & -0.073 & -0.095 & 0.028 & 1.000 & & & \\
\hline RDW & $-0.177^{*}$ & $-0.367^{\ddagger}$ & 0.112 & $-0.289^{\ddagger}$ & $-0.387^{\ddagger}$ & $-0.408^{\ddagger}$ & $-0.192^{*}$ & -0.002 & -0.046 & 1.000 & & \\
\hline GRA & -0.041 & $-0.211^{*}$ & $0.358^{\ddagger}$ & -0.090 & -0.117 & $-0.176^{*}$ & -0.092 & $0.191^{*}$ & -0.048 & 0.101 & 1.000 & \\
\hline MON & 0.135 & $0.277^{\dagger}$ & -0.001 & $0.216^{*}$ & $0.294^{\ddagger}$ & $0.299^{\ddagger}$ & 0.162 & -0.055 & -0.069 & $-0.176^{*}$ & $-0.176^{*}$ & 1.000 \\
\hline LYM & 0.008 & 0.141 & $-0.367^{\ddagger}$ & 0.035 & 0.044 & 0.101 & 0.058 & $-0.181^{*}$ & 0.057 & -0.057 & $-0.961^{\ddagger}$ & -0.065 \\
\hline
\end{tabular}

Significance: ${ }^{*} p<0.05,{ }^{\dagger} p<0.01$, and ${ }^{*} p<0.001$.

$\mathrm{BAZ}=\mathrm{BMI}$-for-age $z$-score, $\mathrm{Hb}=$ hemoglobin, $\mathrm{RBC}=$ red blood cell, $\mathrm{HCT}=$ hematocrit, $\mathrm{MCV}=$ mean corpuscular volume, $\mathrm{MCH}=$ mean corpuscular hemoglobin, $\mathrm{MCHC}=$ mean corpuscular hemoglobin concentration, $\mathrm{RDW}=$ red cell distribution width, WBC = white blood cell, GRA = granulocyte, $\mathrm{MON}$ = monocyte, $\mathrm{LYM}=$ lymphocyte, THR = thrombocyte.

with WBC, RDW, and granulocyte. WBC was negatively correlated with HCT and lymphocyte and positively correlated with thrombocyte and granulocyte. RBC was positively correlated with HCT and monocyte and inversely correlated with MCH and RDW. Similarly HCT was well correlated with $\mathrm{MCV}$ and monocyte and negatively correlated with RDW. $\mathrm{MCV}$ was found to be associated with $\mathrm{MCH}$ and monocyte and negatively correlated with RDW and granulocyte. RDW is negatively correlated with $\mathrm{MCH}$ and monocyte. Granulocyte was positively correlated with $\mathrm{MCHC}$ and negatively correlated with monocyte and lymphocyte. Monocyte was negatively correlated with MCHC.

\section{Discussion}

The present study attempted to assess the association of hematological indices with the prevalence of anemia among children in the rural surroundings of Bhubaneswar city, India. The mean $\mathrm{Hb}$ among school age boys was significantly lower than girls. Sahu et al. [12] also found a lower mean $\mathrm{Hb}$ level in school age boys than girls in Gajapati district, Odisha. Bulliyya et al. [13] showed a mean $\mathrm{Hb}$ level of 10.07 among adolescent girls of Khurda district, Odisha.

The prevalence of anemia among preschool children was $48.5 \%$, which is much less when compared to the state 
data of $92.4 \%$ [14]. The type of anemia among school age children was $68.9 \%$ (mild $27.4 \%$, moderate $23.6 \%$, and severe $17.9 \%)$. Sahu et al. [12] found severity of anemia (35.2\% mild, $59.4 \%$ moderate, and $5.4 \%$ severe) in children in Gajapati district much higher than the value in this study. Girls were significantly more anemic than boys in the age group of 1112 years while more boys were anemic in the age group of 10-11 years. Similar results were reported for school children in Bangalore where prevalence of anemia was higher in boys aged 10 years whereas it was high in girls aged 11 years [15].

The mean HCT, MCV, and MCH of school age boys were significantly lower than girls'. Zemel et al. [16] observed a significantly lower HCT among boys than girls of school age sickle cell children (excluding children receiving transfusion therapy). In this study chronic undernutrition (stunting) may be one of the factors for lower level of HCT. Kokore et al. [17] found that $\mathrm{MCV}$ and $\mathrm{MCH}$ are statistically higher for girls than their male counterparts aged 5-11 years. The hypochromasia (MCH deficient) and microcytosis (MCV deficient) in school age population are higher in boys than in girls. The disruption of erythrocyte parameters like MCV and $\mathrm{MCH}$ precedes the final stage of anemia with concurrent fall in Hb levels below the limit. In this study, decrease in MCV and $\mathrm{MCH}$ might indicate a deficiency in micronutrients including iron and vitamins as suggested earlier [18].

Mean MCV and $\mathrm{MCH}$ were significantly higher among school age girls than preschool girls. Moreover, $\mathrm{MCH}$ and $\mathrm{MCHC}$ of preschool children were low compared to school children. Similar findings were observed among girls of different age groups [19]. It was found that MCV and MCH were slightly lower in those under-5 children but subsequently increased and reached to the adult level by age of 6 years [20]. Several studies reported an increase in mean $\mathrm{MCH}$ and MCHC levels with increase in age $[19,21]$. Vitamin $B_{12}$ deficiency in terms of value above reference level for MCV and $\mathrm{MCH}$ was found to be $14.4 \%$ and $5.7 \%$, respectively. Bleyere et al. [19] reported $5.1 \%$ of probable vitamin $\mathrm{B}_{12}$ deficiency (high MCV) among children.

In the current study, mean WBC count and proportion above the upper level were higher among preschool boys than school age boys (13.8\% versus $5.9 \%)$. Excess WBC in the peripheral blood may be indicative of various disease states, including inflammation (acute or chronic) from bacteria virus or parasites [22]. Porniammongkol et al. [23] demonstrated that the percentage of children with elevated $\mathrm{WBC}$ compared to normal range was higher in the younger age than in the older age. In this study, the prevalence of WBC deficiency was $35.3 \%$ indicating immunosuppression/viral infection while $12.2 \%$ of them had WBC level above the cut-off level representing inflammation or infection. Bleyere et al. [19] found WBC level below the range in $26.8 \%$ and $0.8 \%$ in above the range of children in West Africa. The percentage of children above the upper limit was much higher in this study population and the reason may be that both WBC and granulocyte are inversely correlated with $\mathrm{Hb}(p<$ $0.001)$. It was also previously well documented that WBC and percentage of neutrophil are inversely associated with $\mathrm{Hb}$ [24]. Since a large proportion of study population is anemic $(62 \%)$ that may lead to overall elevated levels of WBC.
The mean RDW was $11.5 \%$ in the study population of which preschool girls have significantly higher level of RDW both by mean level and frequency. It was previously demonstrated that RDW levels were significantly higher in lower age group of iron deficient children in Turkey as well as girls who had higher value than boys [25]. A higher cut-off value for RDW indicates an iron/vitamin $\mathrm{B}_{12}$ /folate deficiency observed to be $11.5 \%$ in the study population. An elevated RDW is also believed to be an early indicator of iron deficiency [26].

The mean values of granulocyte, monocyte, and lymphocyte were $36.71 \%, 5.68 \%$, and $57.38 \%$, respectively, and preschoolers had correspondingly $36.16 \%, 6.02 \%$, and $57.14 \%$ as confirmed by our previous study [27] along with school age children having $37.00 \%, 5.50 \%$, and $57.50 \%$, respectively. The mean monocyte and lymphocyte values in children of West Africa were found to be $5.2 \%$ and $51.3 \%$, respectively [17], which is closer to our value. The prevalence of granulocyte, monocyte, and lymphocyte deficiency was $64.9 \%, 32.8 \%$, and $0.8 \%$, respectively. About $74.8 \%$ of children had high lymphocyte count representing their susceptibility to viral infection. Kokore et al. [17] found only $0.3 \%$ of lymphocyte deficiency in children, which is much less than that of our value, while a high lymphocyte count was observed among $88.7 \%$ of children. In this study chronic infection was indicated by high monocyte count among $6.9 \%$ of children. The prevalence of low monocytes is reported to be less at $6.5 \%$ and $4.8 \%$, respectively, in other studies $[17,19]$. Monocytes represent a source of proinflammatory cytokines and thus are believed to play a role in obesity-associated disease [28]. Chapman et al. [29] demonstrated monocyte concentration to be an independent risk factor for subclinical carotid atherosclerosis. About $63.4 \%$ of children were below the lower range for thrombocyte. Higher level of thrombocytes observed in $7.6 \%$ of children indicates viral infection/pernicious anemia. This high number of platelet deficiency may be due to various cut-off levels suggested to be used for different age group of children whereas many authors recommended to consider a single cut-off for all age groups $\left(<150\right.$ thousand $\left./ \mathrm{mm}^{3}\right)$. Using this range, Kokore et al. [17] and Bleyere et al. [19] found low thrombocytes in $1.9 \%$ and $5.8 \%$ of total population, respectively. It was observed that disorders of the bone marrow and other medical conditions could cause an elevated platelet count [30].

$\mathrm{Hb}$ was positively correlated with RBC, HCT, MCV, $\mathrm{MCH}$, and monocyte in this population. At birth, the total $\mathrm{Hb}$ level, RBC, and HCT are shown to be higher than at any other period of life [31]. The Hb content and the RBCs then gradually rise to adult levels by the age of puberty [32]. Maude et al. [33] even also found RBC correlated positively with total $\mathrm{Hb}$ in homozygous sickle cell patients where there is abnormal synthesis of $\mathrm{Hb}$. It was established that the HCT usually correlates well with $\mathrm{Hb}$ but is even less sensitive for iron deficiency than $\mathrm{Hb}$ [34]. The positive association between $\mathrm{Hb}$ and MCV suggests a lesser chance of macrocytic anemia in the study population as the concentration of $\mathrm{Hb}$ varies concomitantly with cell volume. When RBCs divide in the bone marrow compartment, the resultant two daughter 
cells after each division are slightly smaller than the parent cell. The reduction in the number of such divisions results in the eventual erythrocytes being larger than usual or macrocytic, with a raised MCV leading to an overall reduction in cell division and in a reduction in Hb biosynthesis [35]. Khan et al. [36] found significant relationship between $\mathrm{Hb}$ and $\mathrm{MCH}$ in elderly Pakistani males. Under iron deficiency condition, formation of $\mathrm{Hb}$ is reduced resulting in a reduction of $\mathrm{MCH}$ [37]. The transmembrane protein (ferroportin) is responsible for the transfer of iron from enterocytes and monocytes/macrophages to the circulation [38]. It was found that ferroportin mRNA expression was significantly reduced in monocytes of anemic subjects compared with controls [39]. Importantly, the decreased expression of ferroportin was paralleled by increased iron storage in monocytes of anemia of chronic disease patients as estimated by hyperferritinemia. As a functional consequence of decreased ferroportin expression and the subsequent reduction of cellular iron export, intracellular iron levels will increase which interferes in the process of erythropoiesis, thus decreasing expression of monocytes leading to decrease in $\mathrm{Hb}$.

In this study, RDW was negatively correlated with $\mathrm{Hb}$, RBC count, $\mathrm{HCT}, \mathrm{MCV}, \mathrm{MCH}$, and monocyte. Lippi et al. [40] also found RDW negatively correlated with $\mathrm{Hb}$ and MCV, while inverse relationship of RDW was seen with the $\mathrm{Hb}$ in iron deficiency anemia; however, no such correlation happened with noniron deficiency anemia [41]. RDW is shown to have weak inverse correlation with that of HCT, $\mathrm{Hb}$, and MCV in animal model [42] and humans [43]. The RDW is usually increased in macrocytosis. Occasionally in spherocytosis and polychromatic macrocytes there are small red cell agglutinates or red cells that have been ingested by monocytes [44]. Increased RDW indicates the presence of anisocytosis, which is related to impaired erythropoiesis and erythrocyte degradation, reflecting chronic inflammation and a high level of oxidative stress [45].

RBC was associated with HCT and monocyte and inversely with $\mathrm{MCH}$. In anemia, a reduction of the $\mathrm{Hb}$ is usually accompanied by reduction in the RBC and HCT [44]. It was revealed that venous HCT values correlated highly with circulating RBC volume [46]. Under autologous experimental conditions, the presence of oxidative stressed erythrocytes in blood exacerbates cytokine production markedly and thus the activation status of human monocytes indicates a probable influence of oxidative stress in these children [47]. In anemic condition a marked fall in $\mathrm{RBC}, \mathrm{Hb}$, and HCT and a parallel increase in the MCV and $\mathrm{MCH}$ were observed [44].

HCT was positively correlated with MCV and monocyte. Weir and Scott [35] noticed a positive correlation between HCT and MCV among elderly Pakistani males. MCV was found to be associated with $\mathrm{MCH}$ and monocyte positively and negatively with the granulocyte. There is a strong association between MCV and MCH in iron deficiency and megaloblastic conditions [48]. It was observed that association between high MCV (above cut-off) and high MCH (above cut-off) as well as a higher peripheral blood monocyte count with venous thrombosis indicates association of these blood indices in vivo [49].
WBC was negatively correlated with HCT and lymphocyte and positively with thrombocyte and granulocyte. It is established that HCT among children of 2-16 years is significantly lower among cases of bacterial and viral infection where WBC is higher than controls [24]. WBC was correlated with thrombocyte in the adolescent population [50]. Jabeen et al. [51] also noticed negative correlation of WBC with lymphocyte but positive correlation with granulocyte. Granulocyte was correlated negatively with monocyte and lymphocyte in tandem with others [51]. MCHC was inversely associated with monocyte and positively with granulocyte. The correlation between HCT and monocyte $(p<0.001)$ is more significant than the correlation between $\mathrm{Hb}$ and monocyte $(p<0.01)$. As MCHC is the ratio between $\mathrm{Hb}$ and HCT, negative correlation with monocyte indicates that with increase in monocyte percentage there is increase in $\mathrm{Hb}$, but the rate of increment is less than that of HCT, which is also evidenced by their strength of association.

School age boys had significantly higher mean levels of ferritin than school age girls. It was observed that, beginning in adolescence, males have higher values of ferritin than females, a trend that persists into late adulthood [52]. The positive correlation of BAZ with $\mathrm{Hb}, \mathrm{RBC}$ count, and $\mathrm{HCT}$ shows that rural children suffer more chronic malnourishment coupled with anemia [53]. Higher RDW is associated with systemic inflammation and undernutrition and represents an integrative measure of the pathological process and hence a negative relation between BAZ and RDW [54].

\section{Conclusion}

The present study showed magnitude of anemia among children, which emphasizes the fact that existence of $62 \%$ anemia in the population is a matter of concern which is also related to undernutrition. The prevalence of high lymphocyte count in the population also indicates viral infection. The prevalence of anemia was higher in lower age group, which was further more due to frequent infections. Girls of preschool age showed a probable iron, vitamin $B_{12}$, or folate deficiency as indicated by high RDW value. Girls of the adolescent age (11-12 years) were more anemic indicating more nutritional requirement with the onset of puberty. However, overall school age boys were found to be suffering from higher level of hypochromasia and microcytic anemia. Since the hematological parameters are interrelated with each other as well as with the gender and age groups, constant monitoring and intervention strategy is needed while providing nutritional supplementation to eradicate anemia. We recommend awareness creation on water and sanitation and nutritional counselling to parents on consumption of iron-rich foods and iron supplementation to prevent anemia among young children with special emphasis on those from low income group and socioeconomic deprived communities.

\section{Competing Interests}

The authors declare that there are no competing interests regarding the publication of this paper. 


\section{Authors' Contributions}

Shuchismita Behera was responsible for collecting the data, laboratory analysis, data management, interpreting the data, and writing the paper. Gandham Bulliyya was responsible for conceiving and designing the study, obtaining ethical approval, analyzing the data, interpreting the data, and critically reviewing the paper.

\section{Acknowledgments}

This work was made possible by support from the Director of RMRC, Bhubaneswar. The authors would like to thank the participating children and family members for their willingness to give the necessary information and blood samples. The authors also thank the local leaders and community health workers for their kind support.

\section{References}

[1] World Health Organization, The World Health Report 2002: Reducing Risks, Promoting Healthy Life, World Health Organization, Geneva, Switzerland, 2002.

[2] L. Allen, B. de Benoist, O. Dary, and R. Hurrell, Guidelines on Food Fortification with Micronutrients, WHO, Geneva, Switzerland, 2006.

[3] R. K. Singh and S. Patra, "Extent of anaemia among preschool children in EAG states, India: a challenge to policy makers," Anemia, vol. 2014, Article ID 868752, 9 pages, 2014.

[4] G. Alvarez-Uria, P. K. Naik, M. Midde, P. S. Yalla, and R. Pakam, "Prevalence and severity of anaemia stratified by age and gender in rural India," Anemia, vol. 2014, Article ID 176182, 5 pages, 2014.

[5] L. Allen and J. Casterline-Sabel, "Prevalence and causes of nutritional anemias," in Nutritional Anemia, U. Ramakrishnan, Ed., pp. 7-21, CRC Press, Boca Raton, Fla, USA, 2001.

[6] S. Attri, N. Sharma, S. Jahagirdar, B. R. Thapa, and R. Prasad, "Erythrocyte metabolism and antioxidant status of patients with Wilson disease with hemolytic anemia," Pediatric Research, vol. 59, no. 4, pp. 593-597, 2006.

[7] WHO Multicentre Growth Reference Study Group, "WHO Child Growth Standards: length/height-for-age, weight-for-age, weight-for-length, weight-for-height and body mass index-forage: methods and development," Tech. Rep., World Health Organization, Geneva, Switzerland, 2006, http://www.who.int/ childgrowth/standards/technical_report/en/.

[8] INACG, Measurement of Iron Status. A Report of the International Nutritional Anaemia Consultative Group (INACG), Nutrition Foundation, New York, NY, USA, 1985.

[9] WHO/UNU/UNICEF, Iron Deficiency Anaemia: Assessment, Prevention and Control, A Guide for Programme Managers, WHO, Geneva, Switzerland, 2001.

[10] Peripheral blood reference ranges adapted from University of Washington Medical Center, 2004, http://www.lymphomation .org/peripheral-blood-ref.pdf.

[11] International Waldenstrom's Macroglobulinebia Foundation (IWMF), Complete Blood Count (CBC), International Waldenstrom's Macroglobulinebia Foundation, Sarasota, Fla, USA, 2014.
[12] T. Sahu, N. C. Sahani, and L. Patnaik, "Childhood anemia-a study in tribal area of Mohana block in Orissa," Indian Journal of Community Medicine, vol. 32, no. 1, pp. 43-45, 2007.

[13] G. Bulliyya, G. Mallick, G. S. Sethy, and S. K. Kar, "Haemoglobin status of non-school going adolescent girls in three districts of Orissa, India," International Journal of Adolescent Medicine and Health, vol. 19, no. 4, pp. 395-406, 2007.

[14] National Nutrition Monitoring Bureau (NNMB), "Prevalence of micronutrient deficiencies," NNMB Technical Report 22, National Institute of Nutrition, Indian Council of Medical Research, Hyderabad, India, 2003.

[15] S. Muthayya, P. Thankachan, M. B. Zimmermann et al., "Low anemia prevalence in school-aged children in Bangalore, South India: possible effect of school health initiatives," European Journal of Clinical Nutrition, vol. 61, no. 7, pp. 865-869, 2007.

[16] B. S. Zemel, D. A. Kawchak, K. Ohene-Frempong, J. I. Schall, and V. A. Stallings, "Effects of delayed pubertal development, nutritional status, and disease severity on longitudinal patterns of growth failure in children with sickle cell disease," Pediatric Research, vol. 61, no. 5, pp. 607-613, 2007.

[17] B. A. Kokore, M. N. Bleyere, L. K. Kouakou, D. Kouassi, and P. A. Yapo, "Haematological status and anaemia prevalence among children aged 5 to 11 years in school canteens in Abidjan (Côte D'ivoire)," Middle East Journal of Scientific Research, vol. 14, no. 4, pp. 461-470, 2013.

[18] M. Ramzan, I. Ali, and A. Salam, "Iron deficiency anemia in school children of Dera Ismail Khan, Pakistan," Pakistan Journal of Nutrition, vol. 8, no. 3, pp. 259-263, 2009.

[19] M. N. Bleyere, A. B. Konan, A. K. Amonkan et al., "Changes in haematological parameters of children aged 5 to 18 years in Abidjan, Côte d'Ivoire," Journal of Physiology and Pathophysiology, vol. 4, no. 2, pp. 11-22, 2013.

[20] A. F. Mohsen, A. El-Hazmi, and S. Warsy, "Normal reference values for hematological parameters, red cell indices, $\mathrm{HbA} 2$ and HbFfrom early childhood through adolescence in Saudis," Annals of Saudi Medicine, vol. 210, no. 3-4, pp. 165-169, 2001.

[21] M. S. Khan, S. Shahnaz, R. Sultana, and I. Ali, "Factors affecting Serum Ferritin level in School going children of District Bannu," Journal of Postgraduate Medical Institute, vol. 26, no. 3, pp. 237241, 2012.

[22] N. Abramson and B. Melton, "Leukocytosis: basics of clinical assessment," American Family Physician, vol. 62, no. 9, pp. 2053 2060, 2000.

[23] O. Porniammongkol, U. Yamborisut, T. Intajak, and P. P. Sirichakwal, "Iron status of hill tribe children and adolescent boys: a cross sectional study at a welfare center in Chiang Mai, Thailand," Pakistan Journal of Nutrition, vol. 10, no. 10, pp. 903909, 2011

[24] A. Ballin, Y. Senecky, U. Rubinstein et al., "Anemia associated with acute infection in children," The Israel Medical Association Journal, vol. 14, no. 8, pp. 484-487, 2012.

[25] F. Akin, E. S. Solak, C. Kilicaslan, S. B. Boke, and S. Arslan, "Iron deficiency anemia among hospitalized children in Konya, Turkey," Anemia, vol. 2013, Article ID 514801, 4 pages, 2013.

[26] F. A. Oski, "Iron deficiency in infancy and childhood," The New England Journal of Medicine, vol. 329, no. 3, pp. 190-193, 1993.

[27] S. Behera, G. Bulliyya, P. G. S. Sethy, and K. Kar, "Influence of antioxidant vitamins on iron and haematological indicators in pre-school children," International Journal of Food Nutritional Sciences, vol. 1, no. 1, pp. 88-98, 2012. 
[28] K. S. Rogacev, C. Ulrich, L. Blömer et al., "Monocyte heterogeneity in obesity and subclinical atherosclerosis," European Heart Journal, vol. 31, no. 3, pp. 369-376, 2010.

[29] C. M. L. Chapman, J. P. Beilby, B. M. McQuillan, P. L. Thompson, and J. Hung, "Monocyte count, but not C-reactive protein or interleukin-6, is an independent risk marker for subclinical carotid atherosclerosis," Stroke, vol. 35, no. 7, pp. 1619-1624, 2004.

[30] T. M. S. John, “Reasons for elevated platelet count," 2014, http:// www.livestrong.com/article/120976-reasons-elevated-plateletcount/.

[31] D. Burman, "Haemoglobin levels in normal infants aged 3 to 24 months, and the effect of iron," Archives of Disease in Childhood, vol. 47, no. 252, pp. 261-271, 1972.

[32] M. R. H. Taylor, C. V. Holland, R. Spencer, J. F. Jackson, G. I. O'Connor, and J. R. O’Donnell, "Haematological reference ranges for schoolchildren," Clinical and Laboratory Haematology, vol. 19, no. 1, pp. 1-15, 1997.

[33] G. H. Maude, R. J. Hayes, and G. R. Serjeant, “The haematology of steady state homozygous sickle cell disease: interrelationships between haematological indices," British Journal of Haematology, vol. 66, no. 4, pp. 549-558, 1987.

[34] H. K. Biesalski and J. G. Erhardt, "Diagnosis of nutritional anemia-laboratory assessment of iron status," in Nutritional Anemia, M. B. Zimmermann and K. Kraemer, Eds., pp. 37-43, Sight and Life Press, Basel, Switzerland, 2007.

[35] D. G. Weir and J. M. Scott, "The biochemical basis of the neuropathy in cobalamin deficiency," Baillière's Clinical Haematology, vol. 8, no. 3, pp. 479-497, 1995.

[36] Z. Khan, M. Nawaz, A. Khan, and U. Bacha, "Hemoglobin, red blood cell count, hematocrit and derived parameters for diagnosing anemia in elderly males," Proceedings of the Pakistan Academy of Sciences, vol. 50, no. 3, pp. 217-226, 2013.

[37] B. Thompson, "Food-based approaches for combating iron deficiency," in Nutritional Anemia, M. B. Zimmermann and K. Kraemer, Eds., pp. 338-358, SIGHT and LIFE Press, Basel, Switzerland, 2007.

[38] A. Donovan, C. A. Lima, J. L. Pinkus et al., "The iron exporter ferroportin/Slc40al is essential for iron homeostasis," Cell Metabolism, vol. 1, no. 3, pp. 191-200, 2005.

[39] I. Theurl, V. Mattle, M. Seifert, M. Mariani, C. Marth, and G. Weiss, "Dysregulated monocyte iron homeostasis and erythropoietin formation in patients with anemia of chronic disease," Blood, vol. 107, no. 10, pp. 4142-4148, 2006.

[40] G. Lippi, F. Sanchis-Gomar, E. Danese, and M. Montagnana, "Association of red blood cell distribution width with plasma lipids in a general population of unselected outpatients," Kardiologia Polska, vol. 71, no. 9, pp. 931-936, 2013.

[41] R. Aulakh, I. Sohi, T. Singh, and N. Kakkar, "Red cell distribution width (RDW) in the diagnosis of iron deficiency with microcytic hypochromic anemia," Indian Journal of Pediatrics, vol. 76, no. 3, pp. 265-267, 2009.

[42] C. Guglielmini, H. Poser, A. D. Pria et al., "Red blood cell distribution width in dogs with chronic degenerative valvular disease," Journal of the American Veterinary Medical Association, vol. 243, no. 6, pp. 858-862, 2013.

[43] A. Vayá, L. Rivera, J. Todolí, J. L. Hernandez, B. Laiz, and J. M. Ricart, "Haematological, biochemical and inflammatory parameters in inactive Behçet's disease. Its association with red blood cell distribution width," Clinical Hemorheology and Microcirculation, vol. 56, no. 4, pp. 319-324, 2014.
[44] B. J. Bain, A Beginner's Guide to Blood Cells: Haematological Findings in Health and Disease, Blackwell, London, UK, 2nd edition, 2003.

[45] L. Ferrucci, J. M. Guralnik, R. C. Woodman et al., "Proinflammatory state and circulating erythropoietin in persons with and without anemia," The American Journal of Medicine, vol. 118, no. 11, pp. 1288.el1-1288.e19, 2005.

[46] D. M. Mock, E. F. Bell, G. L. Lankford, and J. A. Widness, "Hematocrit correlates well with circulating red blood cell volume in very low birth weight infants," Pediatric Research, vol. 50, no. 4, pp. 525-531, 2001.

[47] A. M. Liese, M. Q. Siddiqi, J. H. Siegel, T. Denny, and Z. Spolarics, "Augmented TNF- $\alpha$ and IL-10 production by primed human monocytes following interaction with oxidatively modified autologous erythrocytes," Journal of Leukocyte Biology, vol. 70, no. 2, pp. 289-296, 2001.

[48] R. F. Croft, A. M. Streeter, and B. J. O’neill, "Red cell indices in megaloblastosis and iron deficiency," Pathology, vol. 6, no. 2, pp. 107-117, 1974.

[49] S. M. Rezende, W. M. Lijfering, F. R. Rosendaal, and S. C. Cannegieter, "Hematologic variables and venous thrombosis: red cell distribution width and blood monocyte count are associated with an increased risk," Haematologica, vol. 99, no. 1, pp. 194-200, 2014.

[50] G. S. Tell, R. H. Grimm Jr., O. D. Vellar, and L. Theodorsen, “The relationship of white cell count, platelet count, and hematocrit to cigarette smoking in adolescents: the Oslo Youth Study," Circulation, vol. 72, no. 5, pp. 971-974, 1985.

[51] F. Jabeen, H. A. Rizvi, F. Aziz, and A. Z. Wasti, "Hyperglycemic induced variations in hematological indices in type 2 diabetics," International Journal of Advance Research, vol. 1, no. 8, pp. 322334, 2013.

[52] R. S. Gibson, Principles of Nutritional Assessment, Oxford University Press, Oxford, UK, 2nd edition, 2005.

[53] R. Bhoite and U. Iyer, "Magnitude of malnutrition and iron deficiency anemiaamong rural school children: an appraisal," Asian Journal of Experimental Biology Science, vol. 2, no. 2, pp. 354-361, 2011.

[54] Z. Förhécz, T. Gombos, G. Borgulya, Z. Pozsonyi, Z. Prohászka, and L. Jánoskuti, "Red cell distribution width in heart failure: prediction of clinical events and relationship with markers of ineffective erythropoiesis, inflammation, renal function, and nutritional state," American Heart Journal, vol. 158, no. 4, pp. 659-666, 2009. 


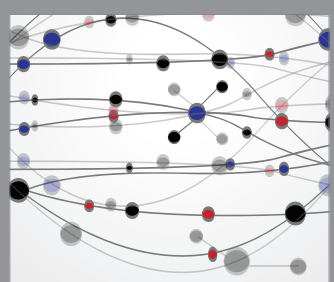

The Scientific World Journal
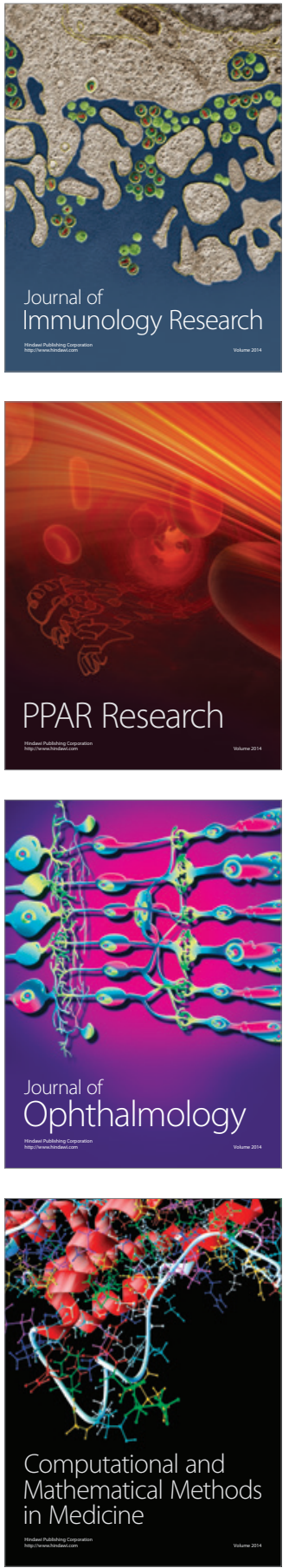

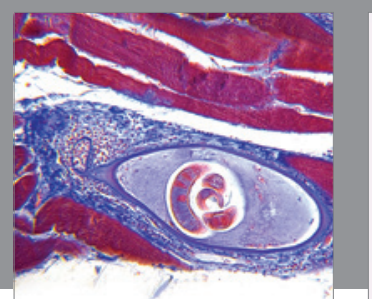

Gastroenterology Research and Practice

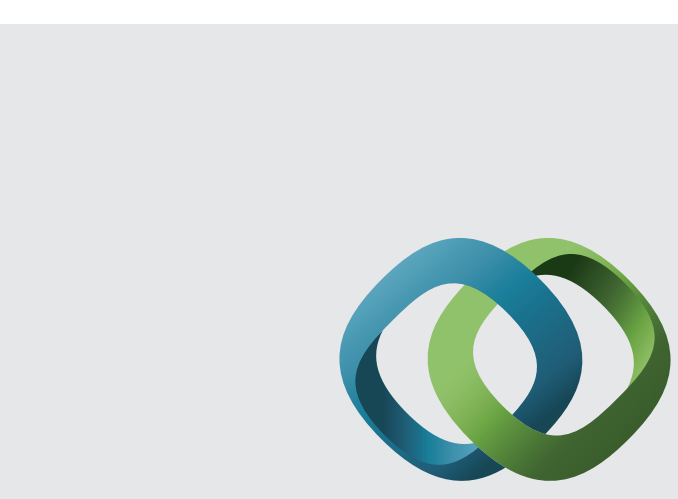

\section{Hindawi}

Submit your manuscripts at

http://www.hindawi.com
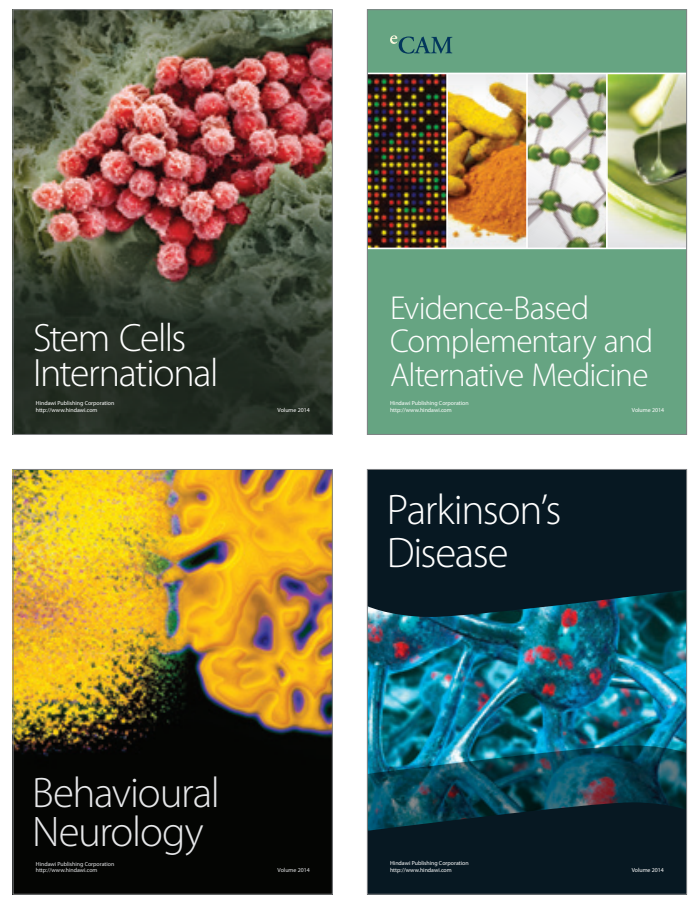
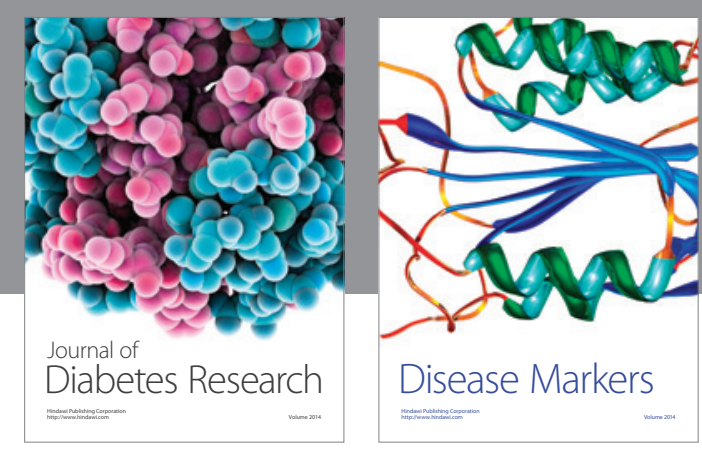

Disease Markers
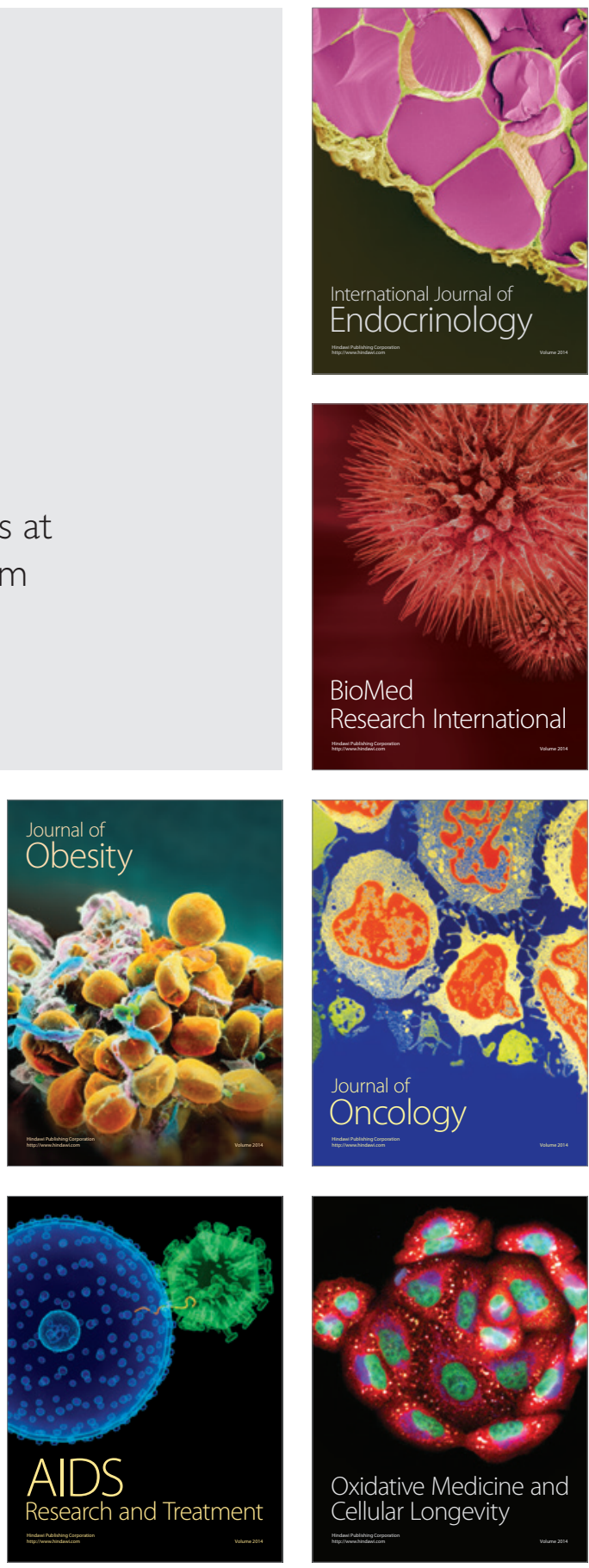